\title{
The Electricity-purchasing Strategy of Power Companies Based on Distributed PV Power Generation Mode
}

\author{
Ying Chen
}

\begin{abstract}
Department of Economic Management, North China Electric Power University, Baoding, 071000, China
\end{abstract}

Keywords: Distributed PV power generation, Electricity-purchasing model, CPSO, Random variables

\begin{abstract}
For the uncertainty of the generating capacity of distributed PV generation caused by meteorological factors, this paper proposes a electricity-purchasing decisions model, taking the generating capacity of distributed PV generation and the price of the spot market as the random variables, using the HPSO algorithm to solve the model. At last, according to the data of purchasing electricity from a region, the model is analyzed and validated. The simulation and comparison show that the model is effective and feasible.
\end{abstract}

\section{Introduction}

PV affected by sunlight great uncertainty for the power company, and its end-user sales price is frozen, how to control a number of uncertainties, avoid market risks, to determine the optimal purchase decisions are most needed to solve the problem. Many experts and scholars on the purchase decision-making issues in-depth research. [1] Based on Nash (Nash) equilibrium model repeated games, to purchase electricity supplier in the electricity market and the proportional distribution contract between the spot market were studied. [2] Based on the structure of bilateral contracts market trading before the market and the market, the establishment of a utility model considering the risks and the unit price of the power suppliers operating constraints of long-term electricity trading. [3] In stochastic programming method for finding the optimal contract capacity optimization strategy.

Documents above the main consideration the uncertainty of demand, purchase questions about uncertainties caused mainly analyzed from a technical point of view. In this paper, the uncertainty of distributed photovoltaic power generation, clean energy a priority to ensure the power supply, the power company proposed to balance the risks and benefits of the purchase decision-making model. And by comparing the PSO algorithm based on chaotic results with actual purchase power company plans to verify the validity and superiority of the model.

\section{Distributed PV Systems}

The installed capacity of distributed PV is usually small, which are dispersedly arranged in the vicinity of users. Distributed PV generally adopt "self-occupied, remaining battery into the grid, using the nearest grid,power line conditioner" operating mode. In this mode, the PV power generation system consists of solar cell matrix, DC power distribution boxes, inverters, and AC power distribution boxes and network equipment configuration, as shown in Fig.1:

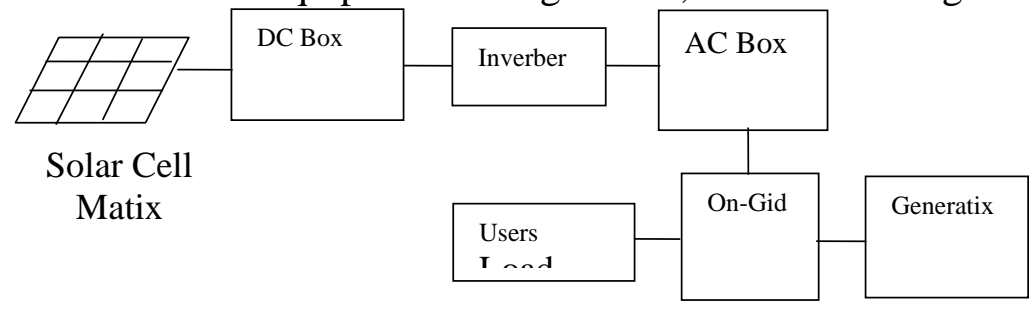

Fig.1 Distributed photovoltaic system 
PV generation converts sunlight into electricity by solar cells. According to the intensity of sunshine and other data , the output power of PV power generation system is calculated as follows:

$$
\begin{gathered}
P=U\left\{I_{\text {scref }}\left[1-M_{1} \exp \left(\frac{U-\Delta U}{M_{2} U_{\text {ocref }}}-1\right)\right]+\Delta I\right\} \\
M_{1}=\left(1-\frac{I_{\text {mref }}}{I_{\text {scref }}}\right) \exp \left(-\frac{U_{\text {mref }}}{M_{2} U_{\text {ocref }}}\right) \\
M_{2}=\left(\frac{U_{\text {mref }}}{U_{\text {ocref }}}-1\right) / \ln \left(1-\frac{I_{\text {mref }}}{I_{\text {scref }}}\right)
\end{gathered}
$$

Where $P, U, I=$ the output power, the output voltage, the output current; $M_{1} 、 M_{2}=$ simplified parameters,; $\Delta U=$ the voltage corrected with the change of conditions; $\Delta I=$ the current corrected with the change of conditions; the exp refers that the base of exponential function is $e$; the ln refers that the base of logarithmic function is $e ; I_{\text {scref }}=$ the short-circuit current; $U_{\text {ocref }}=$ the open-circuit voltage; $U_{\text {mref }}=$ the point voltage of corresponding to the maximum of power; $I_{\text {mref }}=$ the point current of corresponding to the maximum of power;

\section{The Purchasing Models for Administration of Power Supply Based on Distributed PV}

\section{Assumptions}

1)Suppose that the prediction error $a$ is the difference between $G^{p}$ the actual value of the demand of electricity and $G$ the forecasting demand; $a \sim N\left(\mu_{1}, \delta_{1}^{2}\right)$;

2)the prediction error is $c$ the difference between $H^{p}$ the actual value of the total electricity produced by $\mathrm{PV}$ and $H$ the forecasting product, $c \sim N\left(\mu_{2}, \sigma_{2}^{2}\right)$;

3) $r$ = the subsidy of the unit electricity;

\section{The Objective Function}

Power companies buy and sell electricity to obtaine profit are:

$$
\begin{gathered}
R=p_{1} G+r H-p\left(Q-k Q^{p}\right)+k p_{2} Q^{p}+d\left(k Q^{p}-Q\right)^{\prime} \\
\left(k Q^{p}-Q\right)^{\prime}=\max \left(k Q^{p}-Q, 0\right)
\end{gathered}
$$

i)Where $p=$ the spot price; $p_{1}=$ the sales price; $p_{2}=$ the long-term contract price;

ii) $k=$ the proportion of the electricity purchased by long-term contracts;

iii) $d=$ the loss of for remaining electricity from long-term contracts in the spot market;

iv) $Q=G-H ; Q^{p}=G^{p}-H^{p}$;

$$
E(R)=k E(Q)-\left\{E\left(p_{1}\right)\left[E(Q)-k Q^{p}\right]\right\}+C_{o v}\left(p_{1}, Q\right)-d \int_{0}^{k Q^{p}}\left(k Q^{p}-Q\right) d \Phi(Q)+r E(H)(6)
$$

Where $C_{o v}\left(p_{1}, Q\right)=$ the covariance of $p_{1}$ and $Q$;

The optimal quantity of electricity for the administration of power supply is:

$$
Q^{*}=\Phi^{-1}\left\{Q^{p}\left[E\left(p_{1}\right)-p_{2}\right] / d+\Phi(0)\right\}
$$

Where $\phi(x) 、 \Phi(x)=$ the probability density function and the distribution function of $Q$;

\section{Constraints}

Power companies tend to be more sensitive to the risks when obtained the maximize of expected profits,. The constraint considered the level of risk is:

$$
P_{r}\left\{R=k Q-\left[p_{1}\left(Q-k Q^{p}\right)+k p_{2} Q^{p}+d\left(k Q^{p}-Q\right)^{\prime}\right]<\bar{R}\right\} \leq 1-\alpha
$$

Where $\bar{R}$ represents the maximum of profit when power companies ensure the confidence level of $R$ is not less than $\alpha$; 
When the power company makes the purchasing decisions, they preset the maximum of expenditure is $N$.Assuming that the probability that electricity cost is lower than $N$ is not less than $\beta$ :

$$
P_{r}\left\{C=p_{1}\left(Q-k Q^{p}\right)+k p_{2} Q^{p}+d\left(k Q^{p}-Q\right)^{\prime} \leq N\right\} \geq \beta
$$

\section{Solving the Model}

For long-term power-purchasing decisions, it is difficult to use traditional methods to deal with the optimization mode in this paper. So we will use the CPSO to solve it as follows:

1)Let $M=0$, the decision variables are converted into Chaos ones according to the interval conversion formula.we take $\mathrm{V}$ independent random variables as the decision variables according to the probability distribution of $a$ and $c$. They are $a_{1}, a_{2}, \ldots \ldots a_{V}, c_{1}, c_{2}, \ldots \ldots, c_{V}$.

2) According to $c x_{i}^{k}$ obtained from step 1), calculate the next chaotic variable $c x_{i}^{k+1}$ using the Logistic equation [4].

3 ) Change the decision variable ${ }^{c x_{i}^{k+1}}$ into chaos variable $x_{i}^{k+1}$ and get into the objective function to obtain $S_{1}, S_{2}, \ldots \ldots, S_{V}$ according to the following formula:

$$
x_{i}^{k+1}=x_{\min , i}+c x_{i}^{k+1}\left(x_{\max , i}-x_{\min , i}\right), i=1,2, \ldots \ldots, n
$$

4) Take the decision variables as a new solution and evaluate the optimality of it;

5) If the new solution is better than the original solution or the iterations are more than the predefined maximum of iterations, we take the output of this new solution as a chaotic searching results; Otherwise, return to step 2) to continue.

\section{Examples and Analysis}

The data comes from an area for a typical day, The amount of solar radiation that day is $27.1 \mathrm{MJ}$. According to previously given algorithm steps, take $M$ as 30 . The range of inertia weight is $[0.9,0.4]$; Acceleration constant $s_{1}=2, s_{2}=2 ; r_{1}$ and $r_{2}$ are both random variables subjected to uniformly distribution in [0,1]; Take the confidence level as 0.95 . The region have adopted the TOU.

Under the conditions of distributed PV ,solving the model by CPSO, you can get the power company of the proportion of long-term contracts electricity for 24 hours and the spot market price, as shown in Fig.2,Fig.3:

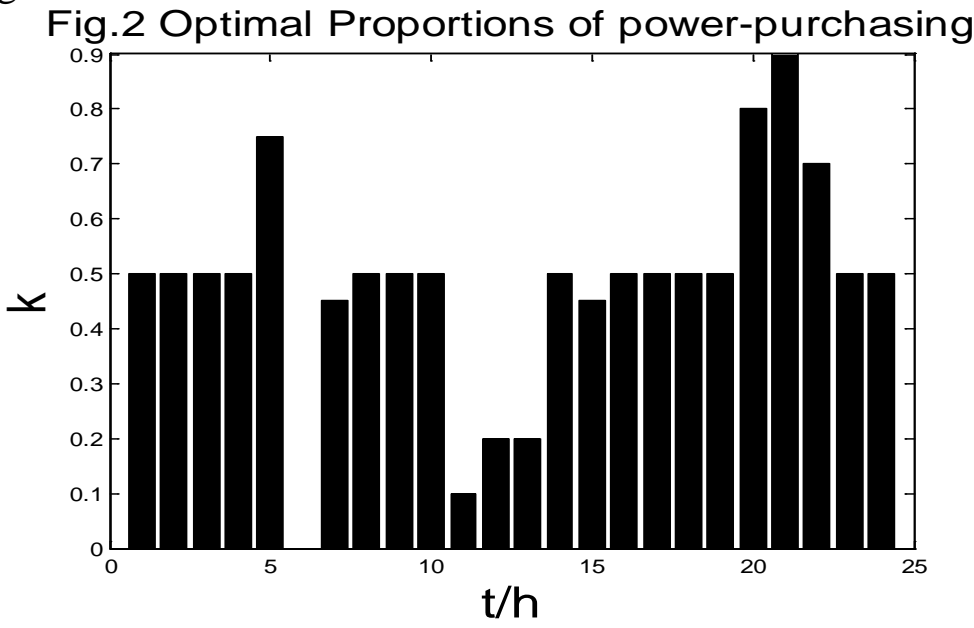




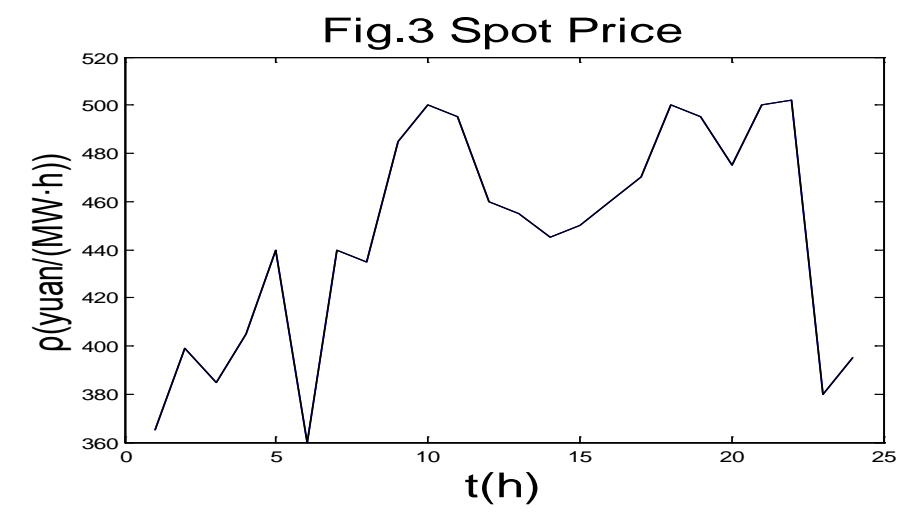

Fig. 4shows the comparison of the power companies profit .The one is calculated by the model and the other is from the actual situation. The results show that it is effective to take risks into consideration for improving the power companies profit.

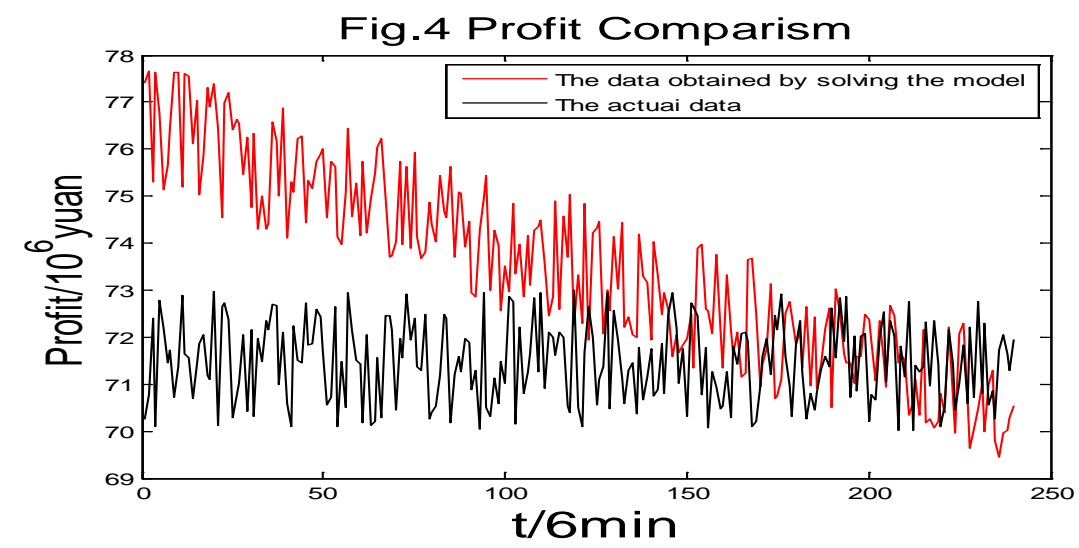

\section{Conclusions}

Provides a stochastic programming based on the sale of electricity distribution companies to purchase unified optimization method. Consider PV great uncertainty and the spot market price and power demand randomness, the power company to maximize profit as the objective function, the risk to the power company can afford to constraints established power companies to purchase power contract and spot market electricity distribution models. Chaos particle swarm algorithm through numerical examples to verify the rationality of the model, the paper discusses the distribution companies tariff changes by the hour, real-time pricing, currently there are some difficulties in the implementation of real-time pricing. However, due to the power user's load flexibility is very small, so the model is provided for research TOU longer time span is also effective.

\section{References}

[1]Yiqun Song, Yixin Ni, Zhijian Hou. Studies on centralized contract generation amount based on equilibrium theory [J]. Proceedings of the CSEE, 2004, 24(7): 64-67.

[2]Xian Zhang, Xifan Wang, Jianxue Wan. A long-term allocating strategy of power generators[J]. Proceedings of the CSEE, 2005, 25(1): 6-12.

[3]Shrestha G B, Pokharel B K, Lie T T. Medium term power planning with bilateral contracts[J]. IEEE Transactions on Power Systems, 2005，20(2): 627-633.

[4]Iba K. Reactive power optimization by genetic algorithm [J]. IEEE Trans on Power Systems, 1994, 9(2): 685-692. 